Research Article

\title{
Automatic Bearing Fault Feature Extraction Method via PFDIC and DBAS
}

\author{
Zhiqiang Liao $\mathbb{D}^{1,2}$ Xuewei Song ${ }^{1},^{2}$ Baozhu Jia $\left(\mathbb{D},{ }^{1}\right.$ and Peng Chen ${ }^{2}$ \\ ${ }^{1}$ Maritime College, Guangdong Ocean University, Zhanjiang, China \\ ${ }^{2}$ Graduate School of Bioresources, Mie University, Tsu, Japan \\ Correspondence should be addressed to Xuewei Song; tianya0218s@126.com and Baozhu Jia; 2501359393@qq.com
}

Received 14 December 2020; Revised 2 April 2021; Accepted 15 May 2021; Published 26 May 2021

Academic Editor: Zhiwei Gao

Copyright (C) 2021 Zhiqiang Liao et al. This is an open access article distributed under the Creative Commons Attribution License, which permits unrestricted use, distribution, and reproduction in any medium, provided the original work is properly cited.

Determining the embedded dimension of a singular value decomposition Hankel matrix and selecting the singular values representing the intrinsic information of fault features are challenging tasks. Given these issues, this work presents a singular value decomposition-based automatic fault feature extraction method that uses the probability-frequency density information criterion (PFDIC) and dual beetle antennae search (DBAS). DBAS employs embedded dimension and singular values as dynamic variables and PFDIC as a two-stage objective to optimize the best parameters. The optimization results work for singular value decomposition for bearing fault feature extraction. The extracted fault signals combined with envelope demodulation can efficiently diagnose bearing faults. The superiority and applicability of the proposed method are validated by simulation signals, engineering signals, and comparison experiments. Results demonstrate that the proposed method can sufficiently extract fault features and accurately diagnose faults.

\section{Introduction}

Bearings are widely applied to rotating machinery equipment, such as motors and pumps. Bearing faults may lead to the destruction of equipment, which in turn results in major downtime, economic losses, and even casualties [1, 2]. Therefore, bearing state requires monitoring and diagnosis.

Bearings usually work in harsh environments, where the background noise is strong and suppresses signal impulses. Meanwhile, the manufacturing errors in the bearing can also generate the background noise $[3,4]$. Therefore, one of the most critical tasks prior to bearing fault diagnosis is to extract fault features from original signals.

Feature extraction is one of the important steps to diagnose the fault by extracting the fault information in the signal as a symptom function for diagnosis. A wide range of fault feature extraction methods have been explored for bearing fault diagnosis. They include filtering methods [5], signal decomposition methods [6], statistical analysis methods [7], and stochastic resonance [8]. An effective feature extraction method is signal decomposition. This method decomposes a signal into several different mode components and reconstructs the signal by extracting the effective modal component that contains sufficient fault information to enhance the fault feature. Examples of such methods include empirical mode decomposition (EMD) and its improved algorithm [9], variational mode decomposition (VMD) [10], local mean decomposition [11], and singular value decomposition (SVD). SVD is particularly effective in fault feature extraction and has excellent stability and invariability. In this method, a singular value (SV) can present a signal's intrinsic characteristics and promote the signal-tonoise ratio (SNR). SVD can extract fault features effectively against strong background noise. The effectiveness of SVD has been proved on the basis of many experiments [12] However, two problems remain: determining the embedded dimension (ED) of the Hankel matrix (HM) and selecting the SV representing the intrinsic features of a fault signal [13]. ED is used to construct the HM, and it plays a crucial role in fault feature extraction results. At the same time, the SVs which represent noise set to 0 represent that the signal is denoised. In some bearing faults, especially in low-speed 
bearing faults, the SNR is low such that the signal energy distribution and background noise are unclear; in this case, successfully selecting the SV is difficult. A number of studies have explored these issues $[14,15]$. Zhao and Ye [16] introduced a method for SV selection based on difference spectra. However, the method's performance decreases when the background noise signal is strong. Gao et al. [14] presented a method for selecting an effective SV through the asymptotic relationship between SVs and vectors. The extracted fault feature signal matrix is reconstructed by minimizing the asymptotic loss and performs better than that resulting from the ordinary reduction of SVs by thresholding methods [17]. However, the method has some assumptions, such as the orthogonal invariance of noise signals. This assumption is difficult to satisfy in engineering projects.

The aforementioned issues imply that existing SVD methods are not ideal in the selection of EDs and SVs. Intelligent optimization methods, such as the genetic algorithm (GA) and particle swarm optimization (PSO), have attracted increasing attention $[18,19]$. These methods can be used to identify the best EDs and SVs. As these optimization algorithms require multiple individuals in the search for optimization solutions, their computational complexity is relatively high. Hence, the current work presents an automatic fault feature extraction method that is based on SVD. Beetle antennae search (BAS) is an efficient optimization method. It enables individual and environmental recognition, it does not need an understanding of function gradient information, and it only requires one individual to search for solutions [20]. Under the designed dual BAS, the ED and SV combination is an associated two-stage objective for optimization. ED is adopted to reconstruct the HM. Each combination based on the selected ED is used to rebuild the extracted fault feature signals. The contributions of this work are as follows: (1) the designed probability-frequency density information criterion (PFDIC) can effectively extract fault features; (2) the proposed algorithm can efficiently select the best ED and SV. According to the designed PFDIC, the extracted signals and normal signals are used to evaluate their similarities. The SV combination with maximum similarity means that the signal contains background noise information. Therefore, the remaining SV is regarded as representative of fault characteristic information and is used for signal reconstruction. The fault feature signal extraction with envelope demodulation can effectively diagnose bearing faults.

The specific contents of this paper are as follows: Section 2 presents the mechanism of SVD fault feature extraction; Section 3 describes the developed method of this study; Sections 4 and 5 show the superiority and applicability of this method with simulation and engineering experiments, respectively; Section 6 describes the comparison experiment; Section 7 presents the conclusions.

\section{SVD-Based Fault Feature Extraction}

This section introduces the mechanism of SVD-based fault feature extraction. Let $\mathbf{X}=\left(x_{1}, x_{2}, \ldots, x_{L}\right)$ be an acquired raw signal of length $L$ in an abnormal state. The signal can construct a $m \times n$ matrix $\mathbf{H}$,

$$
\mathbf{H}=\left[\begin{array}{cccc}
x_{1} & x_{2} & \cdots & x_{n} \\
x_{2} & x_{3} & \cdots & x_{n+1} \\
\vdots & \vdots & \vdots & \vdots \\
x_{m} & x_{m+1} & \cdots & x_{m+n-1}
\end{array}\right],
$$

where $n \geq 2, m \geq 2,1<n<L$, and $m+n-1=L$. Matrix $\mathbf{H} \in R^{m \times n}$. It is called the attractor trajectory matrix or the HM. It has an orthogonal matrix $\mathbf{U} \in R^{m \times m}$ and $\mathbf{V} \in R^{n \times n}$.

$$
\mathbf{H}=\mathbf{U} \boldsymbol{\Sigma} \mathbf{V}^{\mathrm{T}} \text {. }
$$

Equation (2) is the matrix $\mathbf{H}$ of SVD, and $r=\operatorname{rank}(\mathbf{H})$. Here,

$$
\boldsymbol{\Sigma}=\left[\begin{array}{cc}
\boldsymbol{\Sigma}_{1} & 0 \\
0 & 0
\end{array}\right]
$$

where $\Sigma_{1}=\operatorname{diag}\left(\lambda_{1}, \lambda_{2}, \ldots, \lambda_{r}\right), \quad \lambda_{1}, \lambda_{2}, \ldots, \lambda_{r}, \quad$ and $\lambda_{r+1}=\lambda_{r+2}=\cdots=\lambda_{n}=0$ is the SV of matrix $\mathbf{H}$. All nonzero singular values are arranged in the following descending order: $\lambda_{1} \geq \lambda_{2} \geq \cdots \lambda_{i} \geq \cdots \geq \lambda_{r}$. In some situations, the first $i$ th SV mainly reflects the object fault signal, and the smaller SVs reflect the noise signals. The mechanism of SVD-based fault feature extraction is shown in Figure 1.

When SVD performs signal processing, equation (2) can be rewritten as

$$
\mathbf{H}=\mathbf{U} \boldsymbol{\Sigma} \mathbf{V}^{\mathbf{T}}=\sum_{i=1}^{r} \mathbf{A}_{\mathbf{i}}=\sum_{i=1}^{r} \mathbf{u}_{\mathbf{i}} \boldsymbol{\lambda}_{\mathbf{i}} \mathbf{v}_{\mathbf{i}}^{\mathbf{T}},
$$

where $\mathbf{A}_{\mathbf{i}} \in R^{m \times n}, \mathbf{u}_{\mathbf{i}} \in R^{m \times 1}$, and $\mathbf{v}_{\mathbf{i}} \in R^{n \times 1}, i=1,2, \ldots, n$.

Therefore, the SVD operation in the HM can express the original signal as the simple linear superposition of some subsignals. Only by selecting and directly adding SVs representing fault information can fault feature extraction be realized.

\section{Developed Method}

3.1. Beetle Antennae Search. BAS is a type of metaheuristic intelligent optimization method that is based on group optimization [20, 21]. The BAS algorithm imitates the function of antennae and the random walking mechanism of beetles in nature. Beetles use their antennae on both sides of their bodies to find food resources, and their position represents an optimal solution. If one antenna is close to food, then the aromas it picks up will be strong. The beetle then climbs to this position. Therefore, BAS is inspired by the discovery and search behavior of long-horned beetles.

BAS uses food odor intensity at a given position as the value of the objective function. To search the initial uncertain position, a beetle should be able to crawl randomly in all orientations. Vectors with random directions can be constructed as representatives and can be standardized in any dimensional space.

Normalizing the random searching direction is as follows: 


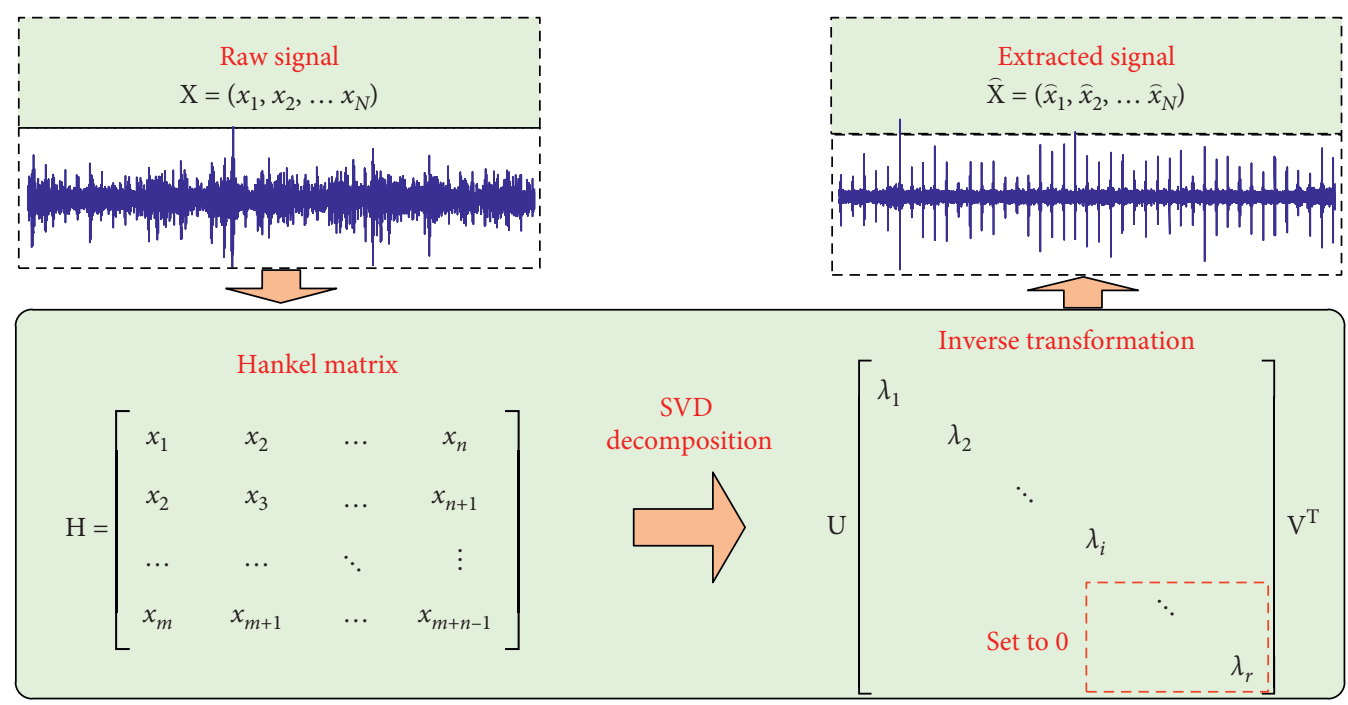

FIGURE 1: SVD-based signal fault feature extraction mechanism.

$$
\vec{b}=\frac{\operatorname{rnd}(\operatorname{dim}, 1)}{\|\operatorname{rnd}(\operatorname{dim}, 1)\|}
$$

Here, $\operatorname{rnd}(\cdot)$ is a random function, and dim denotes the position dimensions.

When a beetle looks for food, it does not know where the food is. It uses two antennae to explore food aromas and climbs toward the direction with the strongest aromas.

The right and left antenna positions are, respectively, obtained as

$$
\begin{aligned}
& x_{r}=x^{t}+d^{t} \cdot \vec{b}, \\
& x_{l}=x^{t}-d^{t} \cdot \vec{b},
\end{aligned}
$$

where $t$ is the iteration number, $x_{r}$ is the right position, $x_{l}$ is the left position, $x^{t}$ is the beetle position, and $d^{t}$ is the antennae's sensing length.

The beetle determines its search behavior on the basis of the detected aromas. Therefore, the beetle's next position is as follows:

$$
x^{t}=x^{t-1}+c \cdot \delta^{t} \cdot \vec{b} \cdot \operatorname{sign}\left(f\left(x_{r}\right)-f\left(x_{l}\right)\right),
$$

where $\delta^{t}$, sign $(\cdot), f(\cdot)$, and $c$ are the searching step size, sign function, optimized function, and the beetle's movement direction, respectively. If the maximum value is optimized, then $c=-1$; otherwise, $c=+1$.

The update of $d^{t}$ and $\delta^{t}$ is

$$
\begin{aligned}
& d^{t}=0.95 d^{t-1}+0.01 \\
& \delta^{t}=0.95 \delta^{t-1}
\end{aligned}
$$

\subsection{Probability-Frequency Density Information Criterion.} In order to perform fault diagnosis accurately, it is necessary to evaluate the similarity between the normal state signal and the abnormal state signal. The PFDIC is used to evaluate the similarities of two signals. The purpose of the PFDIC is to choose the optimal SVs so as to achieve the greatest similarity between reconstructed signals and normal signals. In the design of such a reliable evaluation standard, the higher the similarity of the probability density distribution between reconstructed signals and normal signals, the better the results. The requirements for the similarity of the frequency density distribution are the same as those for the probability density distribution.

The schematic of the PFDIC is shown in Figure 2.

The presented condition should dominate the similarity of the probability density distribution and frequency density distribution.

Given these requirements, the probability density distribution function is designed as follows:

$$
p_{j}=q_{j} \frac{\sqrt{2 \pi}}{2} \int_{0}^{\infty}(2 \pi t)^{2} p_{x}(t) \mathrm{d} t .
$$

Meanwhile, the frequency density distribution function is

$$
q_{j}=\frac{1}{\pi} \sqrt{\frac{\int_{0}^{\infty}(2 \pi t)^{2} P_{x}(t) \mathrm{d} t}{\int_{0}^{\infty} P_{x}(t) \mathrm{d} t} e^{-j^{2} / 2 \sigma_{x}^{2}}} .
$$

According to equations (11) and (12), the PFDIC can be given as

$$
\begin{aligned}
I_{p q} & =I_{p}+I_{q}=\frac{\sum_{j=1}^{n}\left|\log \left\{\left(p_{j}^{*} / p_{j}\right) \times\left(q_{j}^{*} / q_{j}\right)\right\}\right|}{n} \\
& =\frac{\sum_{j=1}^{n}\left|\log \left\{\left(p_{j}^{*} / p_{j}\right)\right\}\right|}{n}+\frac{\sum_{j=1}^{n}\left|\log \left\{\left(q_{j}^{*} / q_{j}\right)\right\}\right|}{n},
\end{aligned}
$$

where $p_{x}(t)$ is the probability density function value at $t, p_{j}^{*}$ and $p_{j}$ are the probability density distribution functions, where $p_{j}^{*}$ represents the reconstructed signals and $p_{j}$ represents the normal signals; $q_{j}^{*}$ and $q_{j}$ are the frequency density distribution functions, where $q_{j}^{*}$ represents the 


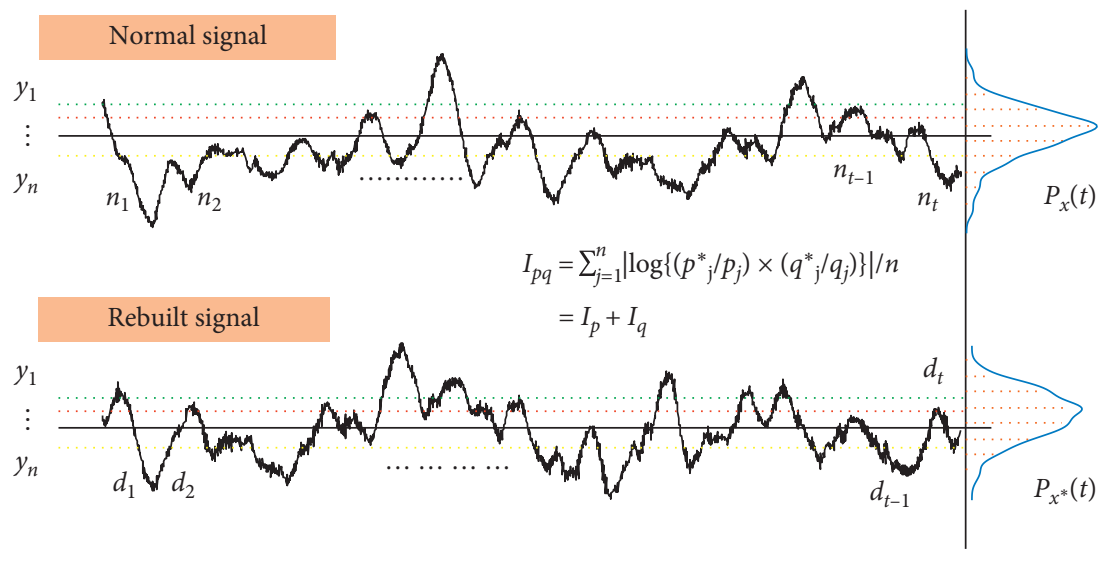

Figure 2: Schematic of PFDIC.

reconstructed signals and $q_{j}$ represents the normal signals; $j$ ranges from 1 to $n$.

When some SVs are selected, the value of $I_{p q}$ is calculated according to equation (13). The smaller the value of $I_{p q}$ is, the higher the similarity will be. At the same time, the selected SV combination represents noise information, and the remaining SVs are considered as the fault information of bearings. The remaining SVs can also extract fault signals.

\subsection{Dual Beetle Antennae Search Based on PFDIC. Dual BAS} is an efficient nonlinear optimization algorithm. It can be used to optimize the parameter optimization in SVD feature extraction. In obtaining the best ED and SVs as a two-stage objective, the dual BAS is designed to optimize the parameters. The first stage is to optimize the ED. Based on the result, the second stage is to optimize the SV combination. The ED is the variable optimization, and the SV combination is the combinatorial optimization. Algorithm 1 shows the detailed steps of the presented method:

3.4. Proposed Fault Signal Extraction Method. Figure 3 shows the flowchart of the method presented in this study. In the first stage of the dual BAS, it generates a random number as the ED and constructs the HM. It decomposes the HM with SVD. In the second stage of dual BAS, BAS is employed to optimize the SV combination.

Taking the PFDIC as the objective function, the BAS can fine-tune the optimal SVs via iterative calculation. The optimal objective value is assigned to the optimal value in the first stage. After the operation, the best ED and SVs can be obtained.

3.5. Bearing Fault Characteristic Frequency and Diagnosis. In the bearing faults of a rolling element, a specific impulse is generated when the rolling element hits the inner race or the outer race; this impulse is called the bearing failure's characteristic frequency. According to [5], $N_{b}$ is the number of rollers, $D_{b}$ is the roller diameter, $D_{c}$ is the bearing pitch diameter, $\alpha$ is the contact angle, and $f_{r}$ is the rotating frequency.
The inner race's ball pass frequency is denoted as BPFI, the outer race's ball pass frequency is denoted as BPFO, and the spin frequency of the roller (ball) is denoted as BSF; they can be, respectively, calculated as follows:

$$
\begin{aligned}
\mathrm{BPFI} & =\frac{N_{b} f_{r}}{2}\left(1+\frac{D_{b} \cos \alpha}{D_{c}}\right), \\
\mathrm{BPFO} & =\frac{N_{b} f_{r}}{2}\left(1-\frac{D_{b} \cos \alpha}{D_{c}}\right), \\
\mathrm{BSF} & =\frac{D_{c} f_{r}}{2 D_{b}}\left(1-\frac{D_{b} \cos \alpha^{2}}{D_{c}}\right) .
\end{aligned}
$$

The extracted fault signal combined with envelope demodulation can calculate the envelope spectrum. Through the comparison of the dominant frequency and fault characteristic frequency, the bearing fault can be effectively diagnosed.

\section{Simulation Experiments}

A local bearing's inner race fault is simulated as follows to analyze the superiority of this study:

$$
\begin{aligned}
& x(t)=m(t) \sum_{-\infty}^{\infty} x_{1}(t) \cdot x_{2}(t) \cdot x_{3}(t)+n(t), \\
& m(t)=A\left(1-B \cos \left(2 \pi f_{r} t\right)\right), \\
& x_{1}(t)=e^{-c\left(t-k T-\tau_{k}\right)}, \\
& x_{2}(t)=\cos \left(2 \pi f_{n}\left(t-k T-\tau_{k}\right)\right), \\
& x_{3}(t)=U\left(t-k T-\tau_{k}\right),
\end{aligned}
$$

where $m(t)$ is the amplitude modulation of the impulse signal, with A set to 4 and $\mathrm{B}$ set to $0.5 ; f_{r}$ is the rotating frequency set to $20 \mathrm{~Hz} ; C$ is the signal damped exponent set to 1,$000 ; f_{n}$ is the resonance frequency set to $5,000 \mathrm{~Hz} . \tau_{k}$ is the tiny fluctuation of the $k$ th impulse relative to feature cycle $T$. The random fluctuation complies with the standard normal distribution, and the standard deviation is $0.5 \%$ of 


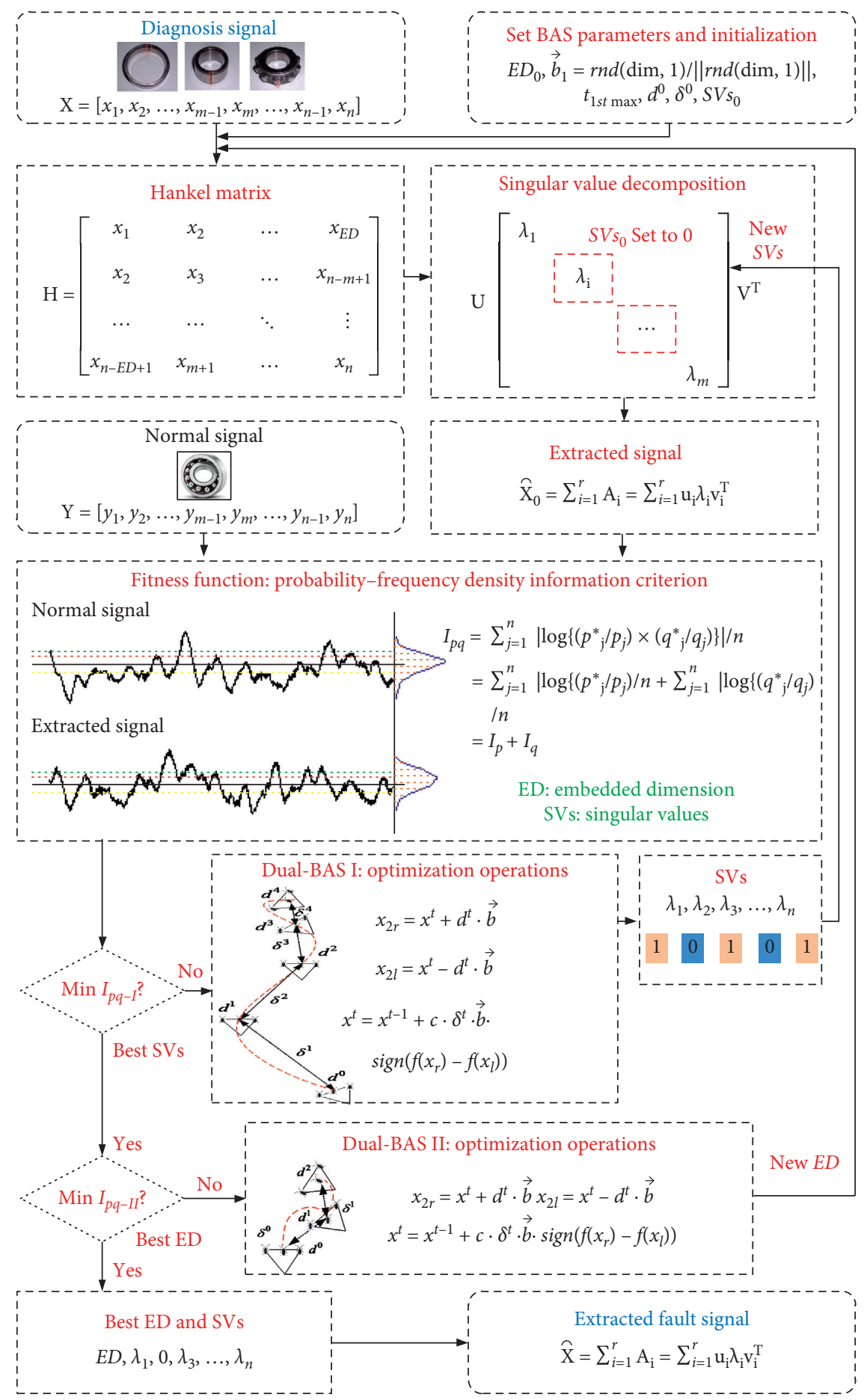

Figure 3: Flowchart of the presented method.

the rotating frequency. The fault characteristic frequency $f_{i}=1 / T$ is $120 \mathrm{~Hz} . U(t)$ is the heavy side function, and $n(t)$ is Gaussian noise with a standard deviation of 2 . Herein, the sampling frequency $f_{s}$ is $12,000 \mathrm{~Hz}$, and the sample length is 4,096 . The simulation signal and its envelope spectrum are shown in Figure 4.

Figure 4(a) shows that, in the simulation signal (red line), the impulse of the signal is conspicuous. Moreover, in the simulation noise signal, the impulse was suppressed by the noise and is thus difficult to identify. Figure 4(b) shows the envelope spectrum of the simulation signal. The fault characteristic frequency is conspicuous, and its harmonics can be established quickly. In Figure 4(c), the fault characteristic frequency cannot be established from the envelope spectrum. Therefore, the simulation noise signal requires fault feature extraction.

According to the designed algorithm, in the first stage of BAS optimization, the ED is used as the optimization 


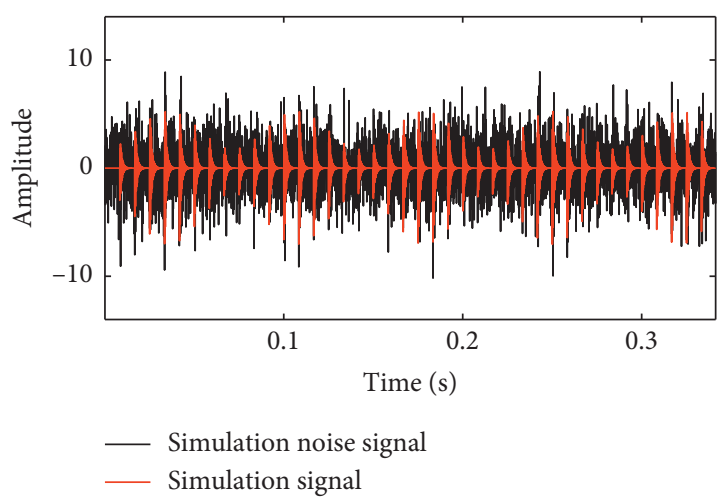

(a)

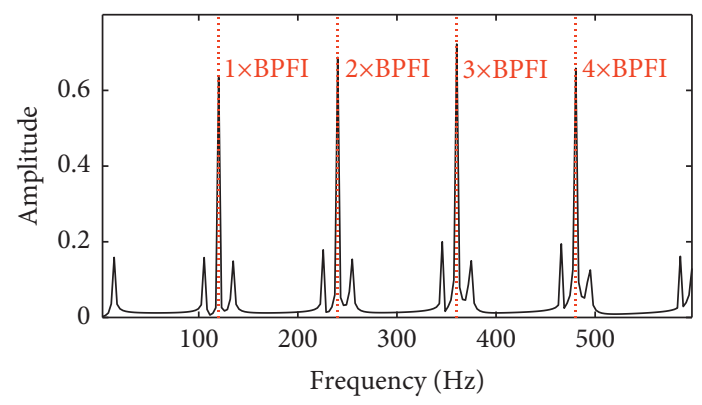

(b)

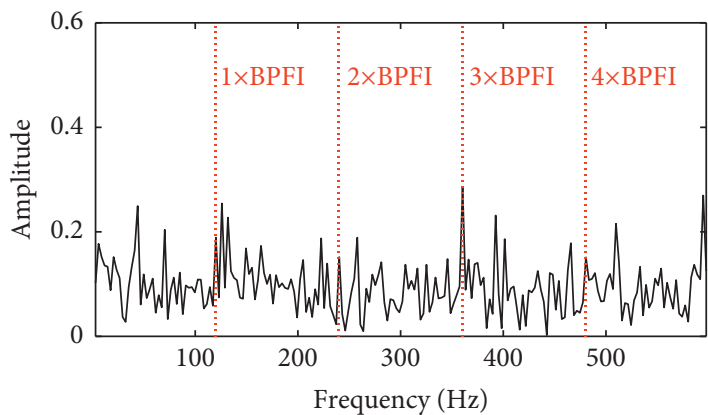

(c)

FIGURE 4: Simulated signal: (a) simulation signal and simulation noise signal, (b) simulation signal's envelope spectrum, and (c) simulation noise signal's envelope spectrum.

variable. Herein, the search range of the $\mathrm{ED}$ is set to $5-200$ (the range given by artificial experience). $d^{0}$ is set to $2, \delta^{0}$ is set to 0.5 , and $r^{0}$ is set to 0.001 . The EDs in the first stage are used as the optimization variables in the second stage of optimization, and the variables are set to 0 or 1 , where 1 denotes the combination of SVs and 0 denotes the noncombination of SVs. The parameters are optimized by the PFDIC. The numbers of optimization iterations in the first and second stages are set to 100 . The objective function after the optimization is shown in Figure 5.

According to the figure of the BSA objective function, the objective value is convergent at generation 13; the best ED is 28; and the best SV combination is 1, 2, 3, 4, and 6. The restructured signal and its enveloped spectrum are shown in Figures 6 and 7.

Figure 7 shows that the restructured signal's dominant frequency and fault characteristic frequency are the same. The fault characteristic frequency and harmonics are conspicuous. The rotating frequency $\mathrm{Fr}$, in addition to the fault characteristic frequency, can also be determined.

To compare the effects of the selected SVs intuitively, this study employs the ED optimized by the proposed method in SVD. Each SV is applied to the reconstructed signal, and kurtosis is applied to evaluate the SNR of the signal. Kurtosis can evaluate the impulse of fault feature extraction. The larger the value is, the better the feature enhancement is. The results are shown in Table 1.

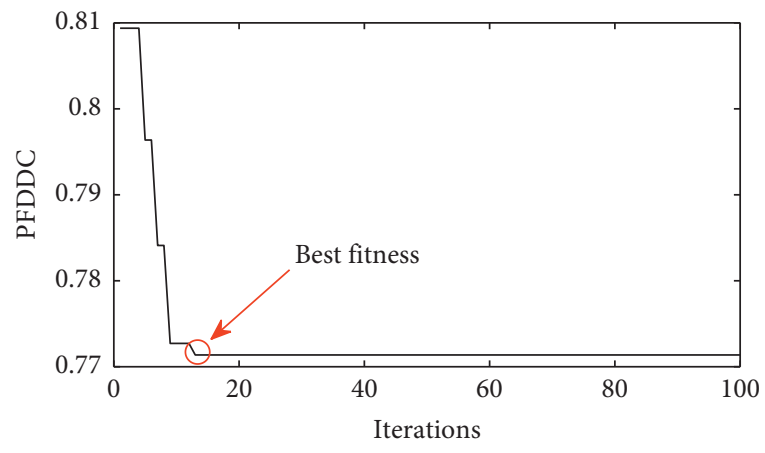

Figure 5: BSA fitness (simulation signal).

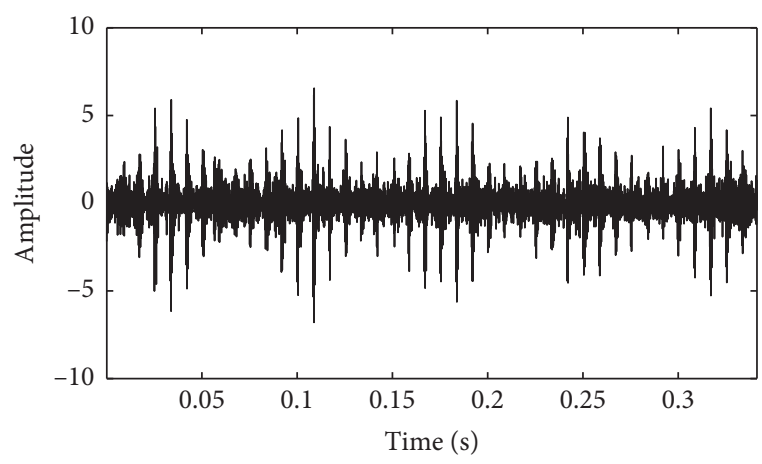

FIGURE 6: Filtered signals. 
(i) Input: Set the maximum number of iterations $t_{1 \text { st max }}, t_{2 \text { nd max }}$. Define the evaluation function $f(\cdot)$ with PFDIC. Randomly set $N$ beetle positions $\left[x_{1 s t i}^{t}, x_{2 \text { ndi }}^{t}\right](i=1,2, \ldots, N)$. Set $t_{1 \text { st }}=0, t_{2 \text { nd }}=0$. Set the value of $c$ on the basis of the optimization purpose. $d^{0}$, $\delta^{0}, x_{\text {best }}, x_{2 \text { nd best }}, g_{\text {best }}\left(\right.$ best objective function in the first stage), $g_{\text {2nd best }}$ (best objective function in the second stage).

(ii) Output: $x_{\text {best }}$ (Best ED, optimal SV combination), $g_{\text {best }}$

(iii) While $\left(t_{1 \mathrm{st}}<t_{1 \mathrm{st} \max }\right)$ or (stop criterion) do

(iv) The first stage of BAS: Generate the direction vector unit $\vec{b}$; update the position $x_{1 \text { str }}^{t_{1 \text { st }}}, x_{1 \text { stl }}^{t_{1 \text { st }}}$. Update the next position $x_{1 \text { st }}^{t_{\text {sst }}+1}$ of the beetle $x_{1 s t}^{t_{\text {st }}}$.

(v) While $\left(t_{2 \text { nd }}<t_{2 \text { nd max }}\right)$ or (stop criterion) do

(vi) The second stage of BAS: Set ED is $x_{1 \text { st }}^{t_{\text {st }}+1}$. Update $x_{2 \text { nd } r}^{t_{\text {nd }}}, x_{2 \text { ndl }}^{t_{\text {nd }}}$, and $x_{2 \text { nd }}^{t_{\text {2nd }}+1}$ of the beetle $x_{2 \text { nd }}^{t_{\text {nd }}}$.

(vii) Calculate the function value $f\left(\left[x_{1 \text { st }}^{t_{\text {st }}+1}, x_{2 \text { nd }}^{t_{\text {nd }}+1}\right]\right)$ of the $i$ th beetle. If $f\left(\left[x_{1 \text { st }}^{t_{\text {st }}+1}, x_{2 \text { nd }}^{t_{\text {2nd }}+1}\right]\right)$ is better than $g_{2 \text { ndbest }}$; $x_{2 \text { ndbest }}=\left[x_{1 \text { st }}^{t_{\text {st }}+1}, x_{2 \text { nd }}^{t_{\text {nd }}+1}\right] ; g_{2 \text { nd best }}=f\left(\left[x_{1 \text { st }}^{t_{\text {st }}+1}, x_{2 \text { nd }}^{t_{\text {nd }}+1}\right]\right) ; t_{2 \text { nd }}=t_{2 \text { nd }}+1$

(viii) End while

(ix) Output the SV combination. Calculate the function value $f\left(\left[x_{1 \text { st }}^{t_{1 \text { st }}+1}, x_{2 \text { nd best }}^{t_{\text {nd }}+1}\right]\right)$ of the $i$ th beetle. If $f\left(\left[x_{1 s t}^{t_{\text {st }}+1}, x_{2 \text { nd best }}^{t_{\text {2nd }}+1}\right]\right)$ is better than $g_{\text {best }}: x_{\text {best }}=\left[x_{1 \text { st }}^{t_{\text {st }}+1}, x_{2 \text { nd }}^{t_{\text {nd }}+1}\right] g_{\text {best }}=f\left(\left[x_{1 \text { st }}^{t_{1 \text { st }}+1}, x_{2 \text { nd best }}^{t_{2 \text { nd }}+1}\right]\right)$.

(x) Update the sensing length of the antennae $d^{t_{1 s t}}$; update the step size of search $\delta^{t_{1 s t}} ; t_{1 \mathrm{st}}=t_{1 \mathrm{st}}+1$

(xi) End while

(xii) Return $x_{\text {best }}, g_{\text {best }}$.

Algorithm 1: Dual BAS based on PFDIC.

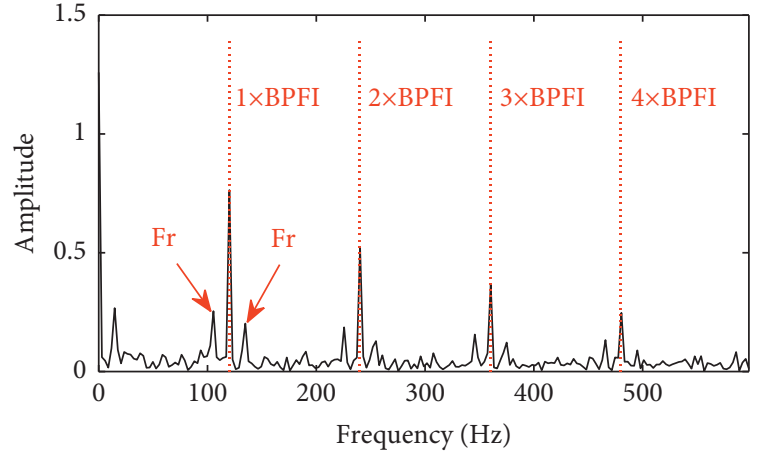

FIgURE 7: Filtered signal envelope spectrum.

As shown in Table 1, the extracted fault feature signal based on dual BAS is effective and advanced.

\section{Engineering Experiment}

5.1. Experiment Platform. The effectiveness of the method is validated by analyzing three vibration fault signals. The experimental platform is set for the condition diagnosis test, and it contains loading equipment, a servo motor, and a motor control system. The platform is shown in Figure 8 .

Four bearing condition signals (normal, outer race fault, inner race fault, and roller fault) are used to analyze the effectiveness of the proposed method. As shown in Figure 8, the bearing is artificially faulted by using electrical discharge machining (fault width: $5.0 \mathrm{~mm}$, depth: $0.6 \mathrm{~mm}$ ). The sampling frequency is $100 \mathrm{kHz}$. The corresponding fault characteristic frequencies are calculated by equations (14)-(16). The results are shown in Table 2.

5.2. Outer Race Fault. The vibration signal is acquired at a rotating speed of $100 \mathrm{rpm}$. The length of the data is 262,144 . Figure 9(a) presents the normal signal and diagnosis signal.
TABLE 1: Results of kurtosis.

\begin{tabular}{lc}
\hline SV no. & Kurtosis \\
\hline 1 & $\mathbf{4 . 6 3 7 2}$ \\
2 & $\mathbf{4 . 8 0 3 7}$ \\
3 & $\mathbf{4 . 5 8 2 4}$ \\
4 & $\mathbf{3 . 8 5 3 5}$ \\
5 & 3.6975 \\
6 & $\mathbf{3 . 9 6 7 6}$ \\
7 & 3.5550 \\
8 & 3.3504 \\
9 & 3.2101 \\
10 & 3.2112 \\
11 & 3.5513 \\
12 & 2.8483 \\
13 & 2.7882 \\
14 & 2.7350 \\
15 & 2.7766 \\
16 & 3.2016 \\
17 & 3.1592 \\
18 & 3.1905 \\
19 & 2.8668 \\
20 & 2.8009 \\
21 & 3.0312 \\
22 & 3.0339 \\
23 & 3.1215 \\
24 & 3.2120 \\
26 & 3.0674 \\
27 & 3.8130 \\
28 & 3.0119 \\
\hline
\end{tabular}

No obvious impulse is noted in the time domain signal. Hence, the impulse signals are suppressed by noise. In the envelope spectrum, the dominant frequency generated by the fault is completely overwhelmed with noise.

With dual BAS, the original signal is filtered, and the envelope is demodulated. On the basis of the programmed convergence, the smaller the parameter is, the better the generation is. After testing, the range of the ED is 15-300. 


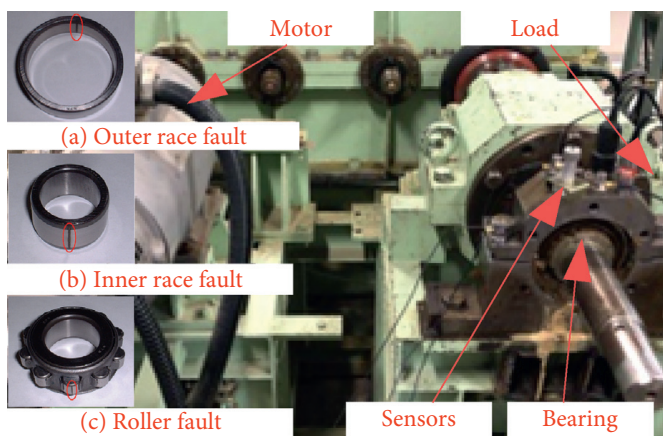

Figure 8: Experimental system for bearing fault diagnosis and bearing faults.

TABLE 2: Bearing fault characteristic frequencies.

Balls: 12 Contact angle: 0

Pitch diameter: $87.5 \mathrm{~mm}$

Ball diameter: $18 \mathrm{~mm}$

RPM: 100

Fault characteristic frequency

\begin{tabular}{lc}
\hline Outer race fault & $7.94 \mathrm{~Hz}$ \\
Inner race fault & $12.06 \mathrm{~Hz}$ \\
Roller fault & $7.76 \mathrm{~Hz}$ \\
\hline
\end{tabular}

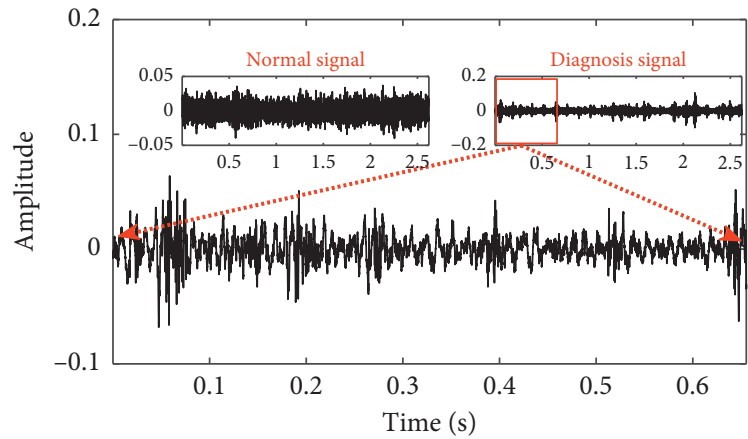

(a)

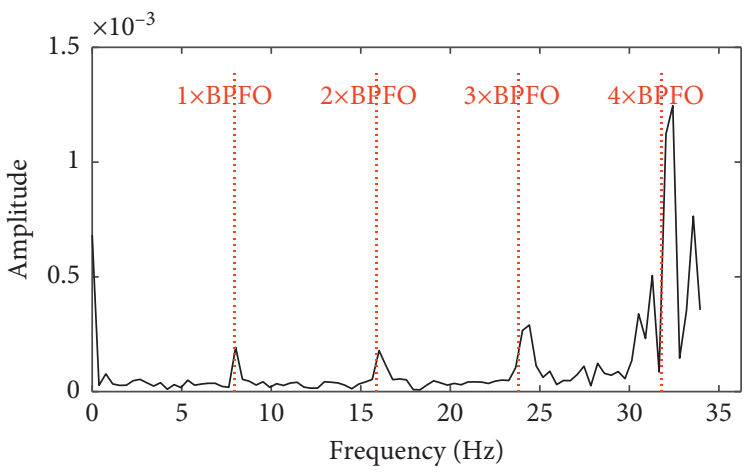

(b)

Figure 9: Outer race fault: (a) time domain; (b) envelope spectrum.

The generation is set to 100 . The BSA fitness, filtered signal, and corresponding envelope spectrum are given in Figures 10 and 11 .

Figure 10 shows the BSA fitness. The value is convergent at generation 4; the best ED is 43; and the best SV combination is $2,3,4,9,10$, and 11. Figure 11 shows the extracted fault feature signal and its envelope spectrum.

From the time domain signal, the impulse signal is conspicuous, and the noise in the extracted fault feature signal is considerably low. As shown in Figure 11(b), the extracted fault feature signal's dominant frequency is $8.0 \mathrm{~Hz}$, and it is equal to the characteristic frequency of the bearing's outer race fault. At the same time, the second harmonic, third harmonic, and fourth harmonic of the dominant frequency are also equal to the corresponding outer race fault's characteristic frequency. The result proves the effectiveness of the proposed method.

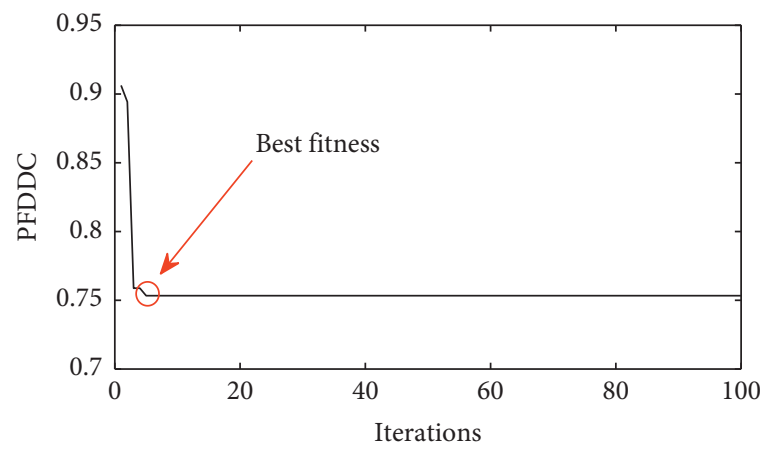

Figure 10: BSA fitness (outer inner fault signal).

5.3. Inner Race Fault and Roller Fault. The superiority of this method is further verified by the inner race fault signal and roller fault signal. The vibration signal is acquired at a 


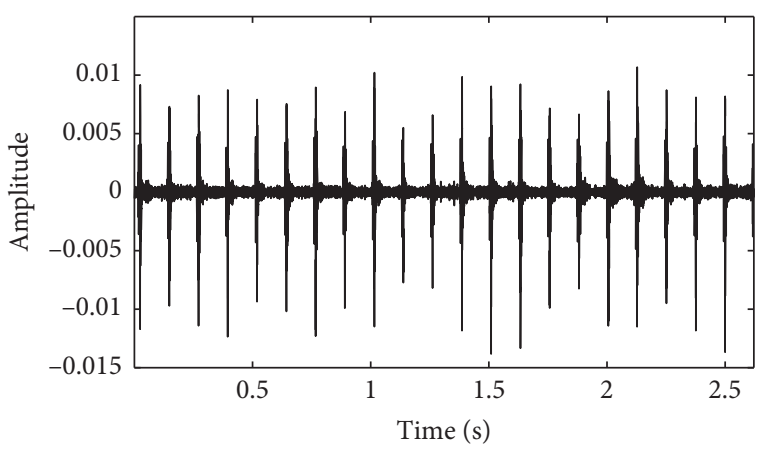

(a)

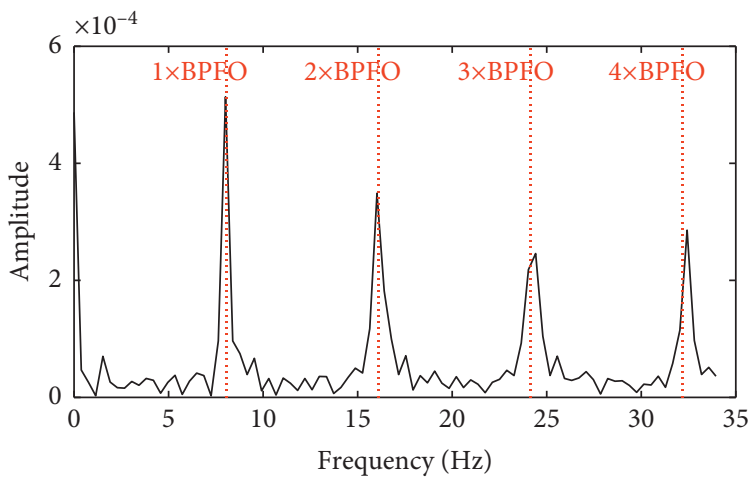

(b)

FIgURE 11: Extracted fault feature signal: (a) time domain; (b) envelope spectrum.

rotating speed of $100 \mathrm{rpm}$. The signal length is 262,144 , and the selected SVs are shown in Figure 12.

The fault feature signal is extracted on the basis of the selected SVs. Figures 13 and 14, respectively, show the original signal envelope spectrum and the envelope spectrum of the extracted fault feature signal of the inner race fault and roller fault.

As shown in Figure 13(b), the extraction fault feature signal's dominant frequency is $12.11 \mathrm{~Hz}$, and it is equal to the characteristic frequency of the bearing's inner race fault. In Figure 14(b), the extraction fault feature signal's dominant frequency is $7.82 \mathrm{~Hz}$, and it is equal to the characteristic frequency of the bearing's roller fault.

These results prove that the proposed method can extract fault features effectively and diagnose faults accurately.

\section{Comparison Experiment}

Comparison experiments are performed to further prove the effectiveness of the proposed method. The comparison experiments include (1) comparison with other SVD methods, (2) comparison with GA and PSO, and (3) comparison with other decomposition methods.

6.1. Comparison with Other SVD Methods. This section compares the proposed method with published methods. The method presented in [22] automatically selects the effective SVs on the basis of the peak of the difference spectrum. In [23], the number of effective SVs was determined using a difference curvature spectrum of incremental singular entropy. In [24], a truncated linear weighting function was proposed to determine the contribution of SVs to the reconstruction of the denoised signal. The presented method is called ISVD. EDs can be determined with the ISVD method. All EDs in the comparison experiments are the same. The authors of $[12,16,24]$ presented algorithm steps in detail. The outer race fault signal is acquired at
$100 \mathrm{rpm}$. The extracted fault feature signals and envelope spectra of the three methods are shown in Figures 15 and 16.

As shown in Figure 16, the proposed method's SV combination is $2,3,4,9,10$, and 11 . The results reveal that all methods can find the fault characteristic frequency. However, the results of the proposed method are the most obvious and have the least noise in the envelope spectrum.

6.2. Comparison with GA and PSO. To prove the effectiveness of the dual BAS, this study designs a comparison experiment with GA and PSO. GA and PSO are relatively mature and intelligent algorithms. They have been used in many fields. Herein, the population numbers of GA and PSO are set to 100, and the other settings are consistent with those in the dual BAS. The convergence result of the objective function is shown in Figure 17.

The convergence speed of dual BAS is better than that of GA but is slightly inferior to that of PSO. However, because BAS involves one population, it takes much less time than GA and PSO. The comparison results are shown in Table 3.

Table 3 shows that the computation times of GA and PSO are similar and that the time used for dual BSA only accounts for about $70 \%$ of that used for GA and PSO. This result proves the effectiveness of the proposed method.

6.3. Comparison with Other Decomposition Methods. A comparison experiment is conducted between the proposed method, EMD method, and VMD method. The experiment uses the outer race fault experiment data. The EMD method and VMD (eight IMF components) method use kurtosis to choose the best IMF. Figure 18 shows the extracted fault feature signal and its envelope spectrum.

From the processed signal, the three methods extract signals with strong impulse. However, the envelope spectrum shows that the presented SVD method has the smallest noise, as shown in the black dotted line block diagram. To quantify the effects of the fault feature signals extracted by 

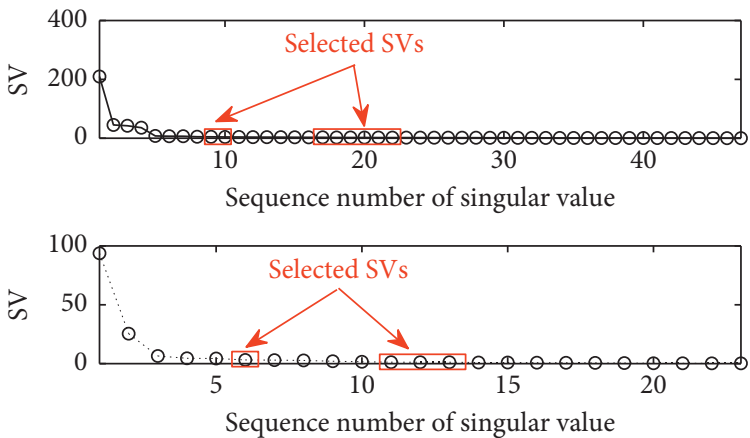

Figure 12: Selected SVs.

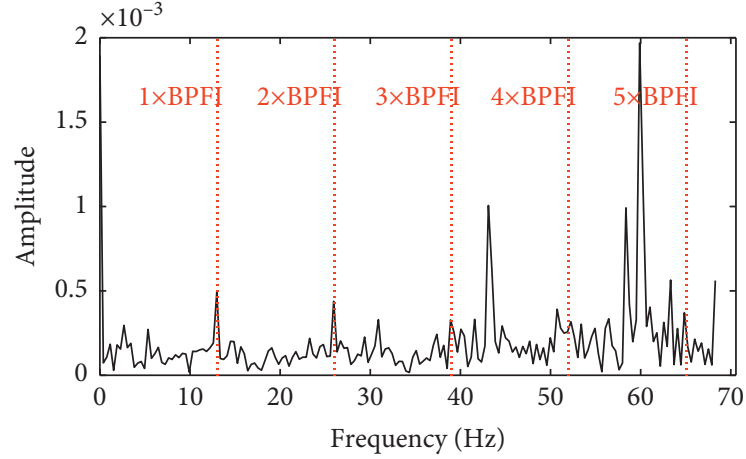

(a)

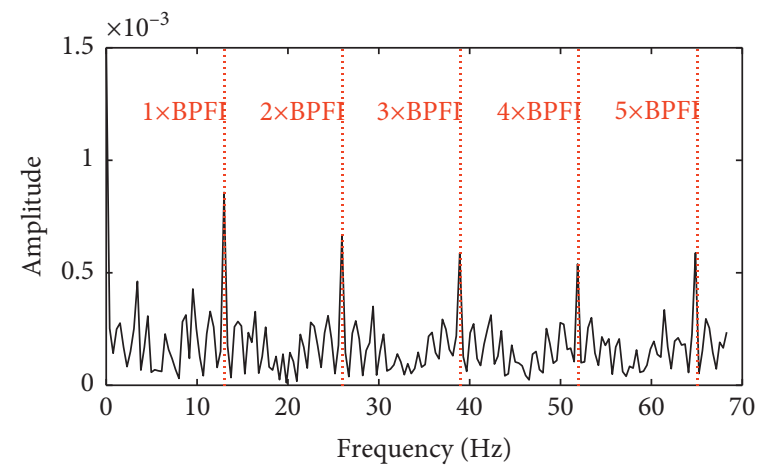

(b)

Figure 13: Inner race fault's envelope spectrum: (a) original signal; (b) extraction fault feature signal.

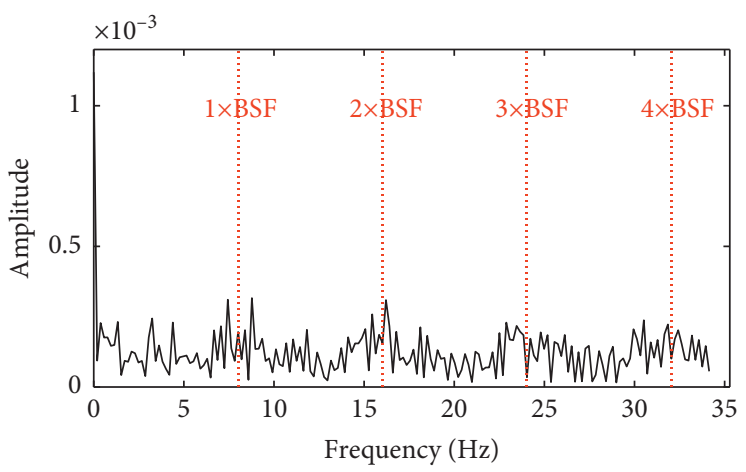

(a)

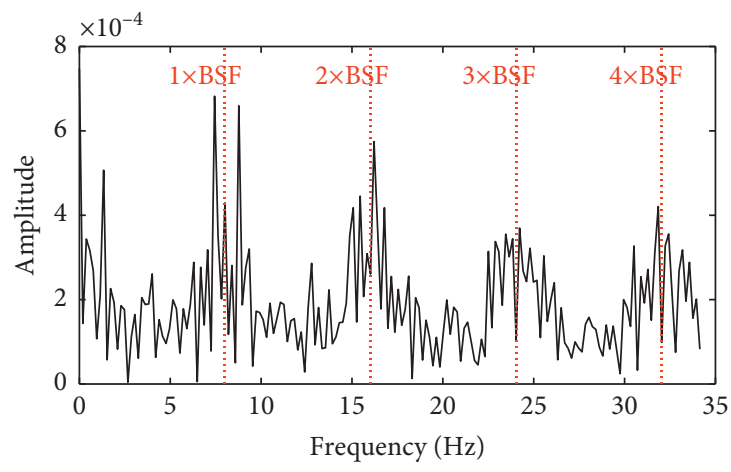

(b)

FIGURE 14: Roller fault's envelope spectrum: (a) original signal; (b) extraction fault feature signal.

the three methods, this study uses the SNR in the evaluation of denoising performance. The SNR is computed as

$$
\mathrm{SNR}(\mathrm{dB})=10 \lg \left(\frac{\sum_{i=1}^{N} x_{i}^{2}}{\sum_{i=1}^{N}\left(x_{i}-\bar{x}_{i}\right)^{2}}\right),
$$

where $x$ is the raw signal, $x$ is the extracted signal, and $N$ is the length of the signal. The larger the SNR value is, the better the denoising effect of the algorithm will be. The SNR results are shown in Table 4.

Table 4 shows that ISVD has the largest SNR value. Moreover, ISVD has the shortest computation time and is 

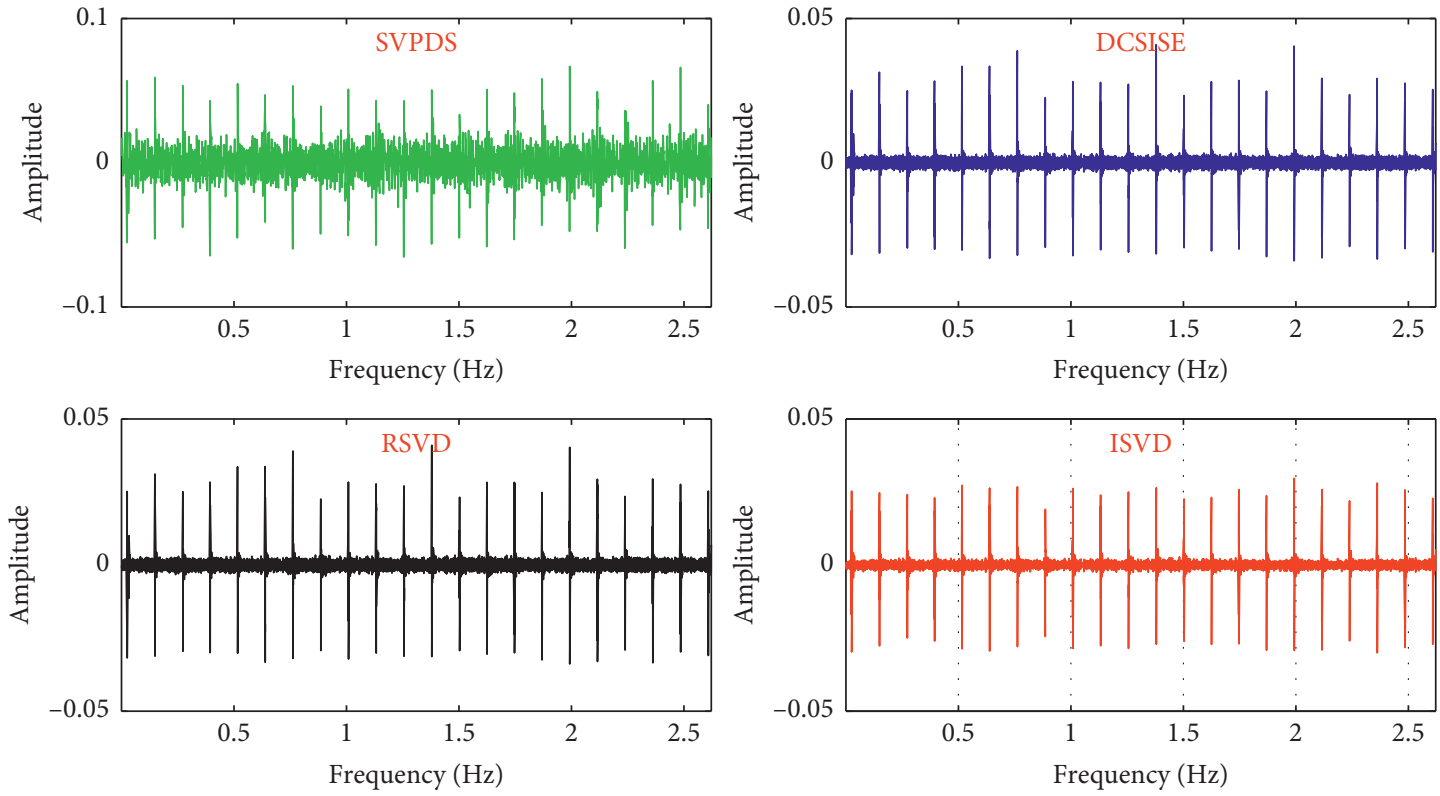

FIGURE 15: Extracted signals of different SVD methods.

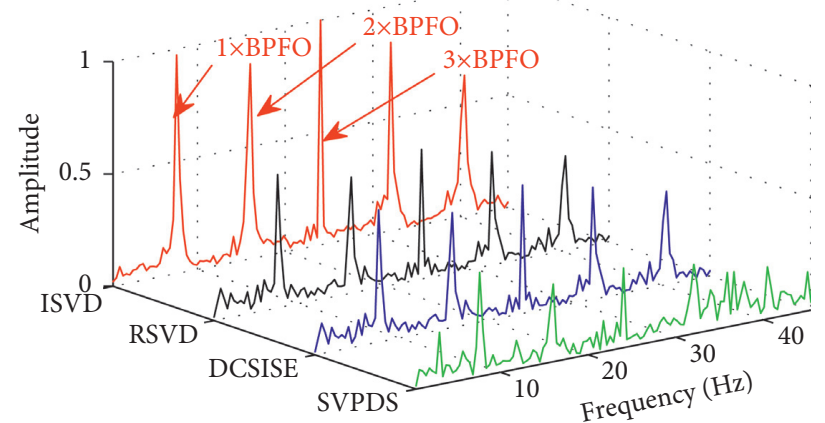

FIGURE 16: Comparison of the proposed method and other SVD methods.

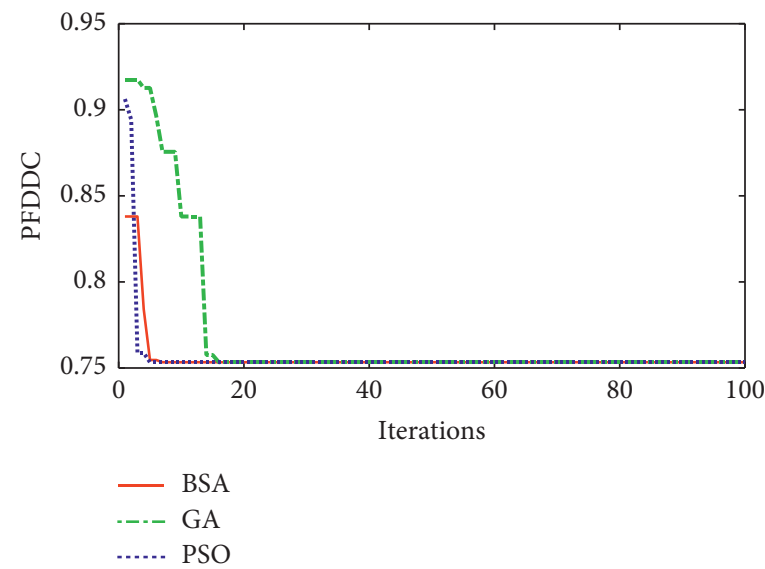

Figure 17: Objective function. 
TABle 3: Optimization results.

\begin{tabular}{lccc}
\hline & BSA & GA & PSO \\
\hline Computation time $(\mathrm{s})$ & 114.345 & 381.136 & 384.136 \\
PFDIC & 0.75331 & 0.75331 & 0.75331 \\
\hline PC & Inter core i5-5200U processor, 64 bit, 8 GB memory, and the MATLAB 2017b \\
\hline
\end{tabular}

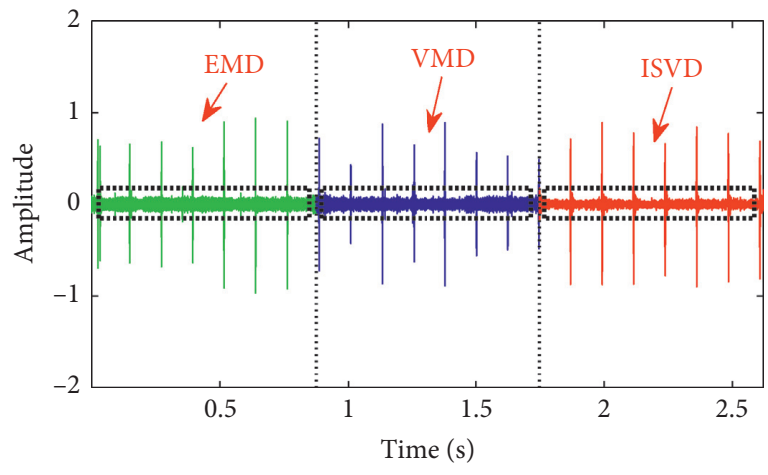

(a)

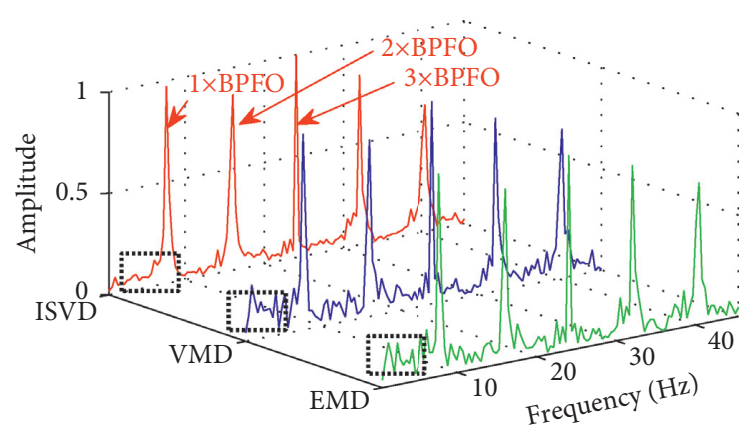

(b)

Figure 18: Results of the decomposition method.

TABLE 4: SNRs and computation times of EMD, VMD, and ISVD.

\begin{tabular}{lcc}
\hline Method & SNR & Computation time $(\mathrm{s})$ \\
\hline EMD & 0.0384 & 363.169 \\
VMD & 0.0047 & 3724.238 \\
ISVD & 1.5399 & 114.345 \\
\hline
\end{tabular}

thus superior to the EMD and VMD methods. This result proves that the method presented in this work can effectively realize bearing fault diagnosis.

\section{Conclusion}

This study proposes an automatic fault feature extraction method based on PFDIC and DBAS to diagnose bearing faults. DBAS employs ED and SVs as dynamic variables while PFDIC, in its two-stage objective, searches the best $\mathrm{HM}$ and selects the SVs. The results indicate that bearing signal SVD can automatically extract fault features. The extracted fault signals combined with envelope demodulation can efficiently diagnose bearing faults. Simulation signals, bearing fault signals, and comparison experiments are constructed to verify the effectiveness and advancement of the presented method. The results show that the proposed method is superior to other methods. In our future work, we will further improve the algorithm's performance and apply it to the fault diagnosis of other rotating machineries.

\section{Data Availability}

The raw/processed data required cannot be shared at this time as the data also form part of an ongoing study.

\section{Conflicts of Interest}

The authors declare that they have no conflicts of interest.

\section{Acknowledgments}

This work was supported by the National Natural Science Foundation of China under Grant no. 52071090. The authors are thankful to Dr. Yao Lizhong, School of Electrical Engineering, Chongqing University of Science and Technology, for his generous support and suggestions to the work.

\section{References}

[1] Z. Gao, S. X. Ding, and C. Cecati, "Real-time fault diagnosis and fault-tolerant control," IEEE Transactions on Industrial Electronics, vol. 62, no. 6, pp. 3752-3756, 2015.

[2] Z. Gao, M. Z. Q. Chen, and D. Zhang, "Special issue on "advances in condition monitoring, optimization and control for complex industrial processes"” Processes, vol. 9, no. 4, p. 664, 2021.

[3] J. Liu, Y. Xu, and G. Pan, "A combined acoustic and dynamic model of a defective ball bearing," Journal of Sound and Vibration, vol. 501, no. 1, Article ID 116029, 2021.

[4] L. Song, H. Wang, and P. Chen, "Automatic patrol and inspection method for machinery diagnosis robot-sound signal based fuzzy search approach," IEEE Sensors Journal, vol. 20, no. 15, pp. 8276-8286, 2020.

[5] Z. Liao, L. Song, P. Chen, and S. Zuo, ““'An automatic filtering method based on an improved genetic algorithm-with application to rolling bearing fault signal extraction," IEEE Sensors Journal, vol. 17, no. 19, pp. 6340-6349, 2017.

[6] W. Huang, N. Li, I. Selesnick et al., "Nonconvex group sparsity signal decomposition via convex optimization for bearing fault diagnosis," IEEE Transactions on Instrumentation and Measurement, vol. 69, no. 7, pp. 4863-4872, 2020.

[7] J. Liu, Z. Xu, L. Zhou, W. Yu, and Y. Shao, "A statistical feature investigation of the spalling propagation assessment for a ball bearing," Mechanism and Machine Theory, vol. 131, pp. 336-350, 2019.

[8] D. Huang, J. Yang, D. Zhou, and G. Litak, "Novel adaptive search method for bearing fault frequency using stochastic resonance quantified by amplitude-domain index," IEEE 
Transactions on Instrumentation and Measurement, vol. 69, no. 1, pp. 109-121, 2020.

[9] H. Wang, Z. Liu, D. Peng, and Y. Qin, “"'Understanding and learning discriminant features based on multiattention 1DCNN for wheelset bearing fault diagnosis," in IEEE Transactions on Industrial Informatics, vol. 16, no. 9, pp. $5735-5745,2020$.

[10] J. Ding, D. Xiao, and X. Li, "Gear fault diagnosis based on genetic mutation particle swarm optimization VMD and probabilistic neural network algorithm," IEEE Access, vol. 8, pp. 18456-18474, 2020.

[11] Q. Yang, L. Chen, Y. Li et al., “An ensemble motor bearing fault diagnosis approach based on LMD feature extraction," in Proceedings of the IEEE International Conference on Signal Processing, Communications and Computing (ICSPCC), pp. 1-5, IEEE, Xiamen, China, October 2017.

[12] M. Zhao and X. Jia, "A novel strategy for signal denoising using reweighted SVD and its applications to weak fault feature enhancement of rotating machinery," Mechanical Systems and Signal Processing, vol. 94, pp. 129-147, 2017.

[13] Z. Liao, L. Song, P. Chen, Z. Guan, Z. Fang, and K. Li, “An effective singular value selection and bearing fault signal filtering diagnosis method based on false nearest neighbors and statistical information criteria," Sensors, vol. 18, no. 7, p. $2235,2018$.

[14] J. Gao, L. Wu, H. Wang, and Y. Guan, "Development of a method for selection of effective singular values in bearing fault signal de-noising," Applied Sciences, vol. 6, no. 5, pp. 154-172, 2016.

[15] D. D. Dai, X. P. Wang, Y. Zhao et al., "Research on denoising ultraviolet spectrum signal with an improved effective singular value selection method," Spectroscopy And Spectral Analysis, vol. 36, no. 7, pp. 2139-2143, 2016.

[16] X. Zhao and B. Ye, "Selection of effective singular values using difference spectrum and its application to fault diagnosis of headstock," Mechanical Systems and Signal Processing, vol. 25, no. 5, pp. 1617-1631, 2011.

[17] A. A. Shabalin and A. B. Nobel, "Reconstruction of a low-rank matrix in the presence of Gaussian noise," Journal of Multivariate Analysis, vol. 118, pp. 67-76, 2013.

[18] X. Song, J. Zhao, J. Song, F. Dong, L. Xu, and J. Zhao, "Local demagnetization fault recognition of permanent magnet synchronous linear motor based on S-transform and PSO-LSSVM," IEEE Transactions on Power Electronics, vol. 35, no. 8, pp. 7816-7825, 2020.

[19] H. Xue, D. Ding, Z. Zhang, M. Wu, and H. Wang, "A fuzzy system of operation safety assessment using multi-model linkage and multi-stage collaboration for in-wheel motor," IEEE Transactions on Fuzzy Systems, vol. 99, p. 1, 2021.

[20] Y. Fan, J. Shao, and G. Sun, "Optimized PID controller based on beetle antennae search algorithm for electro-hydraulic position servo control system," Sensors, vol. 19, no. 12, p. 2727, 2019.

[21] X. Jiang, Z. Lin, S. Li, and S. Ma, "Dynamical attitude configuration with wearable wireless body sensor networks through beetle antennae search strategy," Measurement, vol. 167, pp. 1-20, 2020.

[22] Z. Liao, X. Song, B. Jia, and P. Chen, "Bearing fault feature enhancement and diagnosis based on statistical filtering and 1.5-dimensional symmetric difference analytic energy spectrum," IEEE Sensors Journal, vol. 21, no. 8, pp. 9959-9968, 2021.

[23] X. Song, H. Wang, and P. Chen, "Weighted kurtosis-based VMD and improved frequency-weighted energy operator low-speed bearing-fault diagnosis," Measurement Science and Technology, vol. 32, no. 3, pp. 35016-35027, 2021.

[24] J. Gao, L. F. Wu, H. M. Wang, and Y. Guan, "Development of a method for selection of effective singular values in bearing fault signal de-noising," Applied Sciences-Basel, vol. 6, no. 5, p. 18, 2016. 